\title{
1q21.1 microdeletion identified by chromosomal microarray in a newborn with upper airway obstruction
}

Yoon Hwa Kim ${ }^{1,2}$, Ju Seok Yang ${ }^{1}$, Young Joo Lee ${ }^{1,2}$, Mi Hye Bae ${ }^{2,3}$, Kyung Hee Park ${ }^{2,3}$, Dong Hyung Lee ${ }^{1}$, Kyung-Hwa Shin ${ }^{2,4, *}$, and Seung Chul Kim ${ }^{1,2, *}$

'Department of Obstetrics and Gynecology, Pusan National University School of Medicine, Busan, Korea

${ }^{2}$ Biomedical Research Institute, Pusan National University Hospital, Busan, Korea

${ }^{3}$ Department of Pediatrics, Pusan National University School of Medicine, Busan, Korea

${ }^{4}$ Department of Laboratory Medicine, Pusan National University School of Medicine, Busan, Korea

A 1q21.1 microdeletion is an extremely rare chromosomal abnormality that results in phenotypic diversity and incomplete penetrance. Patients with a 1q21.1 microdeletion exhibit neurological-psychiatric problems, microcephaly, epilepsy, facial dysmorphism, cataract, and thrombocytopenia absent radius syndrome. We reported a neonate with confirmed intrauterine growth restriction (IUGR), micrognathia, glossoptosis, upper airway obstruction, facial dysmorphism, and eye abnormality at birth as well as developmental delay at the age of 1 year. These clinical manifestations, except for the IUGR and upper airway obstruction, in the neonate indicated a 1q21.1 microdeletion. Here, we report a rare case of a 1q21.1 microdeletion obtained via paternal inheritance in a newborn with upper airway obstruction caused by glossoptosis and tracheal stenosis.

Key words: Chromosome deletion, 1q21.1 microdeletion syndrome, Microarray analysis.

\section{Introduction}

A 1q21.1 microdeletion is a rare chromosomal change that is inherited in an autosomal dominant pattern or occurs de novo. The phenotypes of a 1q21.1 microdeletion manifest as various abnormalities such as distinctive facial features, mild to moderate developmental delay, eye problems, and microcephaly. Other findings include genitourinary abnormalities, skeletal malformation, seizure, and hypotonia [1]. Moreover, phenotypes of heart defects, mullerian aplasia, autism, and schizophrenia have also been reported [2-5]. However, no studies have investigated the relationship between upper airway obstruction and 1q21.1 microdeletions. Because of the variability in phenotypic features, a 1q21.1 microdeletion is diagnosed based on chromosomal microarray analysis [6].

Here, we report a rare case of 1q21.1 microdeletion identified by chromosomal microarray from paternal inheritance with upper airway obstruction.

Received: April 3 2018, Revised: May 23 2018, Accepted: May 24 2018, Published: 30 June 2018

*Corresponding author: Seung Chul Kim, M.D., Ph.D. (iD http://orcid.org/0000-0002-8174-9931

Department of Obstetrics and Gynecology, Pusan National University School of Medicine and Biomedical Research Institute, Pusan National University Hospital, 179 Gudeok-ro, Seo-gu, Busan 49241, Korea.

Tel: +82-51-240-7287, Fax: +82-51-248-2384, E-mail: ksch0127@naver.com

Kyung-Hwa Shin, M.D. iD http://orcid.org/0000-0002-8454-4448

Department of Laboratory Medicine and Biomedical Research Institute, Pusan National University Hospital, 179 Gudeok-ro, Seo-gu, Busan 49241, Korea.

Tel: +82-51-240-7463, Fax: +82-51-240-7413, E-mail: skh2009pnuh@gmail.com

Conflict of interest: The authors declare that they do not have any conflicts of interest.

(c) This is an open-access article distributed under the terms of the Creative Commons Attribution Non-Commercial License (http://creativecommons.org/licenses/by-nc/4.0/) which permits unrestricted non-commercial use, distribution, and reproduction in any medium, provided the original work is properly cited.

(c) Copyright 2018 by the Korean Society of Medical Genetics and Genomics 


\section{Case}

A 27-year-old, primigravid woman was referred to our hospital because of hydramnios and intrauterine growth restriction (IUGR) at 35 weeks and 3 days gestation. The prenatal ultrasonogram revealed hydramnios (amniotic fluid index [AFI], 29) along with a small stomach (stomach circumference, $33 \mathrm{~mm}$; stomach circumference/abdominal circumference, 11.2\%) and the expected body weight was 1,944 $\mathrm{g}$ (<3rd percentile). Fetal nuchal translucency was increased $(3.5 \mathrm{~mm})$ at 12 weeks and 1 days gestation, but quadruple markers at 16 weeks and 2 days of gestation were within the normal range. Amniocentesis was performed at 19 weeks of gestation. The results of the karyotype analysis and alpha-fetoprotein level measurements were normal. During a prenatal follow-up, sudden oligohydramnios $(\mathrm{AFI}, 5)$ was detected at 41 weeks of gestation. The neonate was delivered by emergent cesarean delivery because of fetal distress at 41 weeks and 1 days of gestation.

The neonate was a male, weighing 2,630 $\mathrm{g}(<10$ th percentile) at birth with a head circumference of $31 \mathrm{~cm}$ (<10th percentile) and height of $47 \mathrm{~cm}$ (10th to 25th percentile). At delivery, Apgar scores were 5 and 7 at 1 and 5 minutes, respectively. Initially, the mouth of the child was small and facial dysmorphism was suspected. The neonate could not breathe spontaneously, and oxygen saturation was less than 90\%. Immediate intubation was attempted, but endotracheal intubation was not easily achieved. Despite the difficulty of intubation, spontaneous extubation occurred several times. A chest computed tomography scan was performed to evaluate tracheal stenosis, which revealed $1.1 \mathrm{~cm}$ stenosis of the larynx and upper part of the trachea. Therefore, tracheostomy was performed until approximately 30 days of age. Upon evaluation of facial anomalies, the values of the inferior facial and frontal naso-mental angles, which were calculated by fetal magnetic resonance imaging (MRI), were $47^{\circ}$ and $131^{\circ}$, respectively. Therefore, the child was diagnosed with mi-

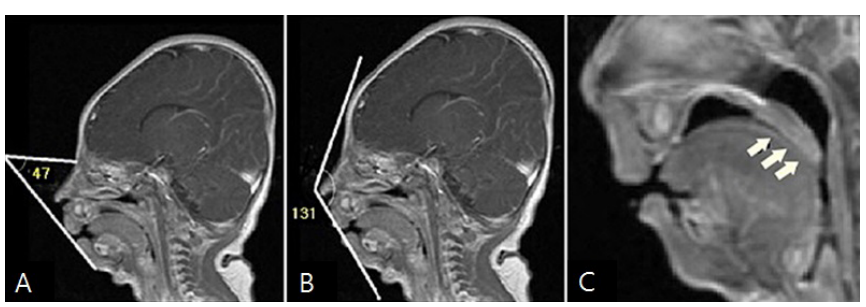

Fig. 1. Magnetic resonance images of a sagittal view of the infant's brain. (A) The inferior facial angle lines showing an angle of $47^{\circ}$. (B) The frontal naso-mental angle lines showing an angle of $131^{\circ}$. (C) Displacement of the tongue base into the oropharynx and the hypopharynx (white arrows). crognathia (Figs. 1A and B). In the sagittal view of the brain MRI, the dorsal part of the tongue compressed the soft palate and induced airway obstruction, and glossoptosis was diagnosed based on MRI findings (Fig. 1C). Therefore, in this case, both glossoptosis and tracheal stenosis were found, which caused the upper airway obstruction. Fundoscopy was performed to evaluate associated ocular anomalies, and persistent hyperplastic vitreous and tractional retinal detachments were diagnosed. Eight days after birth, the infant had a seizure during admission. The neonate was not sucking well and was unable to breastfeed through the bottle, so he was admitted to the Rehabilitation Department. Since then, no seizure-like activity has occurred, but marked dystonia with dyskinesia was confirmed by a rehabilitation doctor. However, there were no specific findings on additional brain MRIs and electroencephalography, nor were any observed in the esophagography and echocardiography.

Standard karyotype and chromosomal microarray analyses were performed to detect genetic disorders associated with a series of abnormal findings. A chromosome 1q21.1 microdeletion of $1.8 \mathrm{Mb}$ was detected by chromosomal microarray analysis (Fig. 2). The patient's mother had undergone surgical repair of ventricular septal defect at the age of 6 years and was administered nifedipine for chronic hypertension. Her chromosomal microarray showed no abnormal findings. The neonate's father is a 28-year-old man with a history of congenital cataract due to unknown cause. The father's chromosomal microarray showed a chromosomal 1q21.1 microdeletion of 1.8 Mb. Evaluation of the father could not be carried out because he was unwilling to participate, but no cognitive impairment or facial dysmorphism was observed.

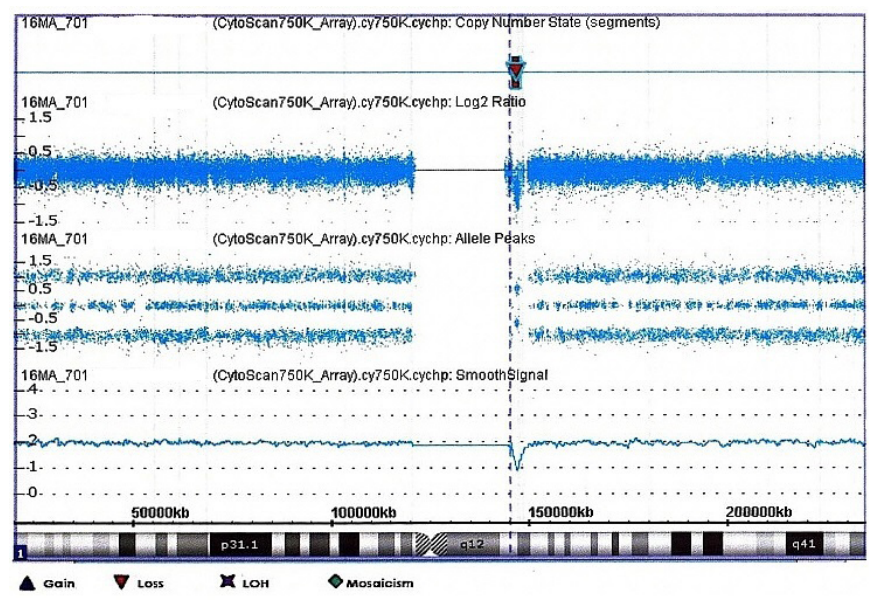

Fig. 2. Chromosomal microarray analysis illustrating a deletion of the chromosomal band 1q21.1 (red arrow, location: 146,023,922 to147,830,830). 
The child showed verbal, motor, and cognitive developmental delay at the age of 1 year according to the Bayley developmental test. Currently, the child is 1 year and 6 months old. He maintains a tracheostomy and feeds through a nasogastric tube. He is undergoing dysphagia therapy and neurodevelopmental treatment in a Rehabilitation Clinic and is being managed with the diagnosis of a 1q21.1 microdeletion.

\section{Discussion}

A 1q21.1 microdeletion is a rare chromosomal abnormality with a phenotype of neurological-psychiatric problems, microcephaly, epilepsy, facial dysmorphism, cataract, and thrombocytopenia absent radius syndrome [7]. In our report, the neonate was confirmed based on his IUGR and small stomach before birth, micrognathia, glossoptosis, facial dysmorphism, upper airway obstruction, eye abnormality at birth, and developmental delay at the age of 1 year. This is the first report of upper airway obstruction in a patient with a 1q21.1 microdeletion. The upper airway obstruction may have been due to both glossoptosis and tracheal stenosis.

A 1q21.1 microdeletion can be inherited in an autosomal dominant manner, and $18-50 \%$ of the cases occur de novo [6]. In general, parents who carry a 1q21.1 microdeletion have milder signs and symptoms than their children who inherit the deletion, although the deletion is the same size. The $1 q 21.1$ microdeletion can be inherited from a parent with minimally abnormal or completely normal clinical findings, even when the proband has severe symptoms. Approximately $25 \%$ of these parents have no associated features [2,6-10]. This suggests that the 1q21.1 microdeletion has reduced penetrance and variable expressivity [6]. In our case, the father showed only cataract, while the child showed more severe symptoms, particularly upper airway obstruction.

In this case, the IUGR and small stomach were observed by prenatal ultrasonogram. Because this case was complicated with hydramnios, it was difficult to properly verify the face anomaly. Microcephaly, facial dysmorphism, and eye problems were confirmed after the child was born. It is important to prepare as much as possible for any predictable concomitant problems that may be encountered during pregnancy or after childbirth. The American College of Obstetricians and Gynecologists recommends prenatal chromosomal microarray analysis for a patient with a fetus with one or more major structural abnormalities identified by ultrasonogram examination [11]. If multiple anomalies are observed in prenatal ultrasonogram, performing a chromosomal microarray in addition to the conventional chromosome test may help to diagnose the genetic disease and suspected syndrome.

In conclusion, upper airway obstruction may occur in patients with a 1q21.1 microdeletion. In the case of a newborn with upper airway obstruction, a trained pediatrician should be prepared for post-delivery airway intervention. Therefore, precise assessments of the fetus suspected as having a genetic syndrome, including a 1q21.1 microdeletion syndrome, are needed during the prenatal and postnatal periods. Appropriate management of the newborn at and after birth can be performed, leading to improved neonatal outcomes.

\section{Acknowledgements}

This study was supported by clinical research grant in 2016 from Pusan National University Hospital.

\section{References}

1. Gamba BF, Zechi-Ceide RM, Kokitsu-Nakata NM, Vendramini-Pittoli S, Rosenberg C, Krepischi Santos AC, et al. Interstitial 1q21.1 microdeletion is associated with severe skeletal anomalies, dysmorphic face and moderate intellectual disability. Mol Syndromol 2016;7:344-8.

2. Christiansen J, Dyck JD, Elyas BG, Lilley M, Bamforth JS, Hicks M, et al. Chromosome 1q21.1 contiguous gene deletion is associated with congenital heart disease. Circ Res 2004;94:1429-35.

3. Cheroki C, Krepischi-Santos AC, Szuhai K, Brenner V, Kim CA, Otto PA et al. Genomic imbalances associated with mullerian aplasia. J Med Genet 2008;45:228-32.

4. Szatmari P, Paterson AD, Zwaigenbaum L, Roberts W, Brian J, Liu XQ, et al. Mapping autism risk loci using genetic linkage and chromosomal rearrangements. Nat Genet 2007;39:319-28.

5. Stefansson $H$, Rujescu $D$, Cichon $S$, Pietiläinen $O P$, Ingason $A$, Steinberg $S$, et al. Large recurrent microdeletions associated with schizophrenia. Nature 2008;455:232-6.

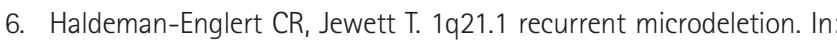
Adam MP, Ardinger HH, Pagon RA, Wallace SE, et al., eds. GeneReviews $^{\circledR}$. Seattle (WA): University of Washington, 1993-2018.

7. Basel-Vanagaite L, Goldberg-Stern $H_{1}$ Mimouni-Bloch $A_{1}$ Shkalim $V_{\text {, }}$ Böhm D, Kohlhase J. An emerging 1q21.1 deletion-associated neurodevelopmental phenotype. J Child Neurol 2011;26:113-6.

8. Shaffer LG, Kashork CD, Saleki R, Rorem E, Sundin K, Ballif BC, et al. Targeted genomic microarray analysis for identification of chromosome abnormalities in 1500 consecutive clinical cases. J Pediatr 2006;149:98-102. 
9. Brunetti-Pierri N, Berg JS, Scaglia F, Belmont J, Bacino CA, Sahoo T, et al. Recurrent reciprocal 1q21.1 deletions and duplications associated with microcephaly or macrocephaly and developmental and behavioral abnormalities. Nat Genet 2008;40:1466-71.

10. Mefford HC, Sharp A, Baker C, Itsara A, Jiang Z, Buysse K, et al. Recurrent rearrangements of chromosome $1 \mathrm{q} 21.1$ and variable pediat- ric phenotypes. N Engl J Med 2008;359:1685-99.

11. Committee on Genetics and the Society for Maternal-Fetal Medicine. Committee opinion No. 682: Microarrays and next-generation sequencing technology: the use of advanced genetic diagnostic tools in obstetrics and gynecology. Obstet Gynecol 2016;128:e262-8. 